\title{
On the disjunctive Markov principle
}

\author{
U. Kohlenbach* \\ Department of Mathematics \\ Technische Universität Darmstadt \\ Schlossgartenstraße 7, 64289 Darmstadt, Germany \\ kohlenbach@mathematik.tu-darmstadt.de
}

May 1, 2015

\begin{abstract}
In this note we show that over a strong (semi-)intuitionistic base theory, the recursive comprehension principle $\Delta_{1}^{0}$-CA does not imply the disjunctive Markov principle $\mathrm{MP}^{\vee}$.
\end{abstract}

Keywords: Law-of-excluded middle, Markov principle, intuitionistic mathematics, realizability.

Mathematics Subject Classification (2010): 03F55, 03F10, 03B20

Consider the following weak forms of the law-of-excluded-middle principle

$$
\mathrm{MP}^{\vee}: \forall \alpha^{1}, \beta^{1}(\underbrace{\left.\begin{array}{c}
\neg(\neg \exists n(\alpha(n) \neq 0) \wedge \neg \exists n(\beta(n) \neq 0)) \\
\rightarrow \neg \neg \exists n(\alpha(n) \neq 0) \vee \neg \neg \exists n(\beta(n) \neq 0)
\end{array}\right)}_{\operatorname{MP}^{\vee}(\alpha, \beta): \equiv}
$$

(introduced in [2] under the name of 'disjunctive Markov principle') and

$$
\Delta_{1}^{0} \text {-LEM : } \forall \alpha, \beta\left(\begin{array}{r}
\forall x(\exists n(\alpha(x, n)=0) \leftrightarrow \forall n(\beta(x, n)=0)) \\
\rightarrow \exists n(\alpha(x, n)=0) \vee \neg \exists n(\alpha(x, n)=0)
\end{array}\right)
$$

(studied e.g. in [1]) as well as the weak comprehension principle

$$
\Delta_{1}^{0} \text {-CA : } \forall \alpha, \beta\left(\begin{array}{c}
\forall x(\exists n(\alpha(x, n)=0) \leftrightarrow \forall n(\beta(x, n)=0)) \\
\rightarrow \exists f^{1} \forall x(f(x)=0 \leftrightarrow \exists n(\alpha(x, n)=0))
\end{array}\right) .
$$

As shown in [2], $\mathrm{MP}^{\vee}$ follows intuitionistically from both the Markov principle MP (for numbers, also called $\Sigma_{1}^{0}$-DNE) as well as from the $\Sigma_{1}^{0}$-LLPO principle (called SEP in [2]). Furthermore, $\Delta_{1}^{0}$-CA trivially implies $\Delta_{1}^{0}$-LEM. For further background information see

${ }^{*}$ The author has been supported by the German Science Foundation (DFG Project KO 1737/5-2). 
$[2,1]$, but note that in the formulations above we allow for function parameters whereas in [1] first-order versions are considered. In the following, $\mathcal{S}^{\omega}$ denotes the full set-theoretic model of E-HA ${ }^{\omega}$ (see [3] to which we also refer for all other undefined notions in this note). We show that, over E-HA ${ }^{\omega}$, the principle $\Delta_{1}^{0}$-CA does not imply $\mathrm{MP}^{\vee}$. In fact this even holds for strong extensions of E-HA ${ }^{\omega}+\Delta_{1}^{0}$-CA. We first observe:

Proposition 1. E-HA ${ }^{\omega}+\mathrm{AC}+\mathrm{IP}_{e f}^{\omega} \vdash \Delta_{1}^{0}$-CA. In fact, instead of $\mathrm{IP}_{e f}^{\omega}$ the weaker principle $\mathrm{IP}_{\forall}^{0}$ suffices.

Proof: By the assumption of $\Delta_{1}^{0}$-CA we, in particular, have

$$
\forall x(\forall m(\beta(x, m)=0) \rightarrow \exists n(\alpha(x, n)=0))
$$

and so by $\operatorname{IP}_{\forall}^{0}$

$$
\forall x \exists n(\forall m(\beta(x, m)=0) \rightarrow \alpha(x, n)=0) .
$$

By AC we get the existence of a function $g$ such that

$$
\forall x(\forall m(\beta(x, m)=0) \rightarrow \alpha(x, g(x))=0) .
$$

One easily show that $f(x):=\alpha(x, g(x))$ satisfies the conclusion of $\Delta_{1}^{0}$-CA.

Theorem 2. Let $\Gamma$ be any set of $\exists$-free axioms which are true in $\mathcal{S}^{\omega}$. Then

$$
\mathrm{E}-\mathrm{HA}^{\omega}+\mathrm{AC}+\mathrm{IP}_{e f}^{\omega}+\Gamma \nvdash \mathrm{MP}^{\vee}
$$

In fact, one can extend $\Gamma$ to also include $\mathcal{S}^{\omega}$-valid axioms whose modified realizability (in $\mathcal{S}^{\omega}$ ) only requires continuous functionals of type degrees $\leq 2$.

The theorem follows using the following two lemmas:

Lemma 3. There is no continuous functional $\varphi: \mathbb{N}^{\mathbb{N}} \times \mathbb{N}^{\mathbb{N}} \rightarrow \mathbb{N}$ such that

$$
\mathcal{S}^{\omega} \models \varphi m r \mathrm{MP}^{\vee},
$$

where $m r$ denotes modified realizability (see e.g. [3]).

\section{Proof:}

$$
\mathcal{S}^{\omega}=\varphi \operatorname{mr} \mathrm{MP}^{\vee}
$$

implies (using classical logic) that

$$
(*) \mathcal{S}^{\omega}=\left\{\begin{array}{l}
\forall \alpha, \beta(\exists n(\alpha(n) \neq 0) \vee \exists n(\beta(n) \neq 0) \\
\rightarrow[\varphi(\alpha, \beta)=0 \rightarrow \exists n(\alpha(n) \neq 0)] \wedge[\varphi(\alpha, \beta) \neq 0 \rightarrow \exists n(\beta(n) \neq 0)]) .
\end{array}\right.
$$

If $\varphi$ is continuous then it is uniformly continuous for $\alpha, \beta \leq 1$, i.e. there exists an $N \in \mathbb{N}$ such that $\varphi(\alpha, \beta)=\varphi\left(\alpha^{\prime}, \beta^{\prime}\right)$ whenever $\bar{\alpha}(N)=\overline{\alpha^{\prime}}(N)$ and $\bar{\beta}(N)=\overline{\beta^{\prime}}(N)$ for all $\alpha, \alpha^{\prime}, \beta, \beta^{\prime} \leq 1$. 
Now consider $\varphi(0,0)$ (where 0 denotes the constant- 0 function).

Case 1: $\varphi(0,0)=0$. Define $\alpha:=\lambda k .0$ and $\beta(n):=0$, if $n \leq N$ and $\beta(n):=1$, if $n>N$. Then $\varphi(\alpha, \beta)=\varphi(0,0)=0$ and $\exists n(\alpha(n) \neq 0) \vee \exists n(\beta(n) \neq 0)$, but $\neg \exists n(\alpha(n) \neq 0)$ contradicting $(*)$.

Case 2: $\varphi(0,0) \neq 0$. Now take $\alpha:=\beta$ (with $\beta$ as in case 1 ) and $\beta:=0$. Again we get a contradiction to $(*)$.

Lemma 4. Let $\Gamma$ be as in Theorem 2. E-HA ${ }^{\omega}+\mathrm{AC}+\mathrm{IP}_{e f}^{\omega}+\Gamma$ has an $m r$-interpretation in E-HA ${ }^{\omega}+\Gamma$ (and hence in $\mathcal{S}^{\omega}$ ) by closed terms of E-HA ${ }^{\omega}$. If $\Gamma$ is extended by further axioms $\Phi$ whose $m r$-interpretation is assumed to be satisfied by new constants $\underline{c}$, then the resulting system has an $m r$-interpretation in E-HA ${ }^{\omega}+\Gamma+(\underline{c} m r \Phi)$ by closed terms built up by the constants of E-HA ${ }^{\omega}$ and $\underline{c}$.

Proof: This follows from [3] (Theorem 5.8) (see also [4]).

Proof of Theorem 2: Suppose that $\mathrm{MP}^{\vee}$ would be derivable in the respective theory, then it would have (valid in $\mathcal{S}^{\omega}$ ) an $m r$-interpretation by a functional $\varphi^{2}$ which is given by a term $t[\underline{c}]$ of E-HA ${ }^{\omega}$ plus extra constants $\underline{c}$ of type level 2 which are interpreted by continuous functionals. Since $t[\underline{c}]$ is primitive recursive (in the sense of Gödel's $T$ ) in $\underline{c}$ and of type 2 it denotes (interpreted in $\mathcal{S}^{\omega}$ ) a continuous functional (to see this one can use e.g. Scarpellini's model $\mathcal{C}^{\omega}=T$ of continuous functionals and $C_{2} \subset S_{2}$, see [3]). This, however, contradicts Lemma 3.

The proof of Theorem 2 above only shows that the full 2 nd order version $\forall \alpha, \beta \operatorname{MP}^{\vee}(\alpha, \beta)$ of $\mathrm{MP}^{\vee}$ is not provable in the respective theory since Lemma 3 crucially requires this. We now show that even for primitive recursive sequences $\alpha_{x}(n):=s(x, n), \beta_{x}(n):=t(x, n)$ of functions $\alpha, \beta$ (s,t primitive recursive terms), $\mathrm{MP}^{\vee}$ is not derivable, i.e. that the function parameter-free version $\mathrm{MP}^{\vee}$ is underivable, using the following

Lemma 5. There is no total recursive function $f: \mathbb{N} \rightarrow \mathbb{N}$ such that

$$
\mathcal{S}^{\omega} \models f m r \forall x^{0} \operatorname{MP}^{\vee}\left(\alpha_{x}, \beta_{x}\right)
$$

for $\alpha_{x}, \beta_{x}$ as defined above for suitable primitive recursive $s, t$.

Proof: Define (using the primitive recursive $T, U$ from the Kleene normal form theorem)

$$
\begin{aligned}
s(x, n): & =\left\{\begin{array}{l}
1, \text { if } T(x, x, n) \wedge U(n)=1, \\
0, \text { otherwise }
\end{array}\right. \text { and } \\
t(x, n): & =\left\{\begin{array}{l}
1, \text { if } T(x, x, n) \wedge U(n)=0, \\
0, \text { otherwise. }
\end{array}\right.
\end{aligned}
$$

Then

$$
\exists n\left(\alpha_{x}(n) \neq 0\right) \leftrightarrow\{x\}(x) \simeq 1, \exists n\left(\beta_{x}(n) \neq 0\right) \leftrightarrow\{x\}(x) \simeq 0 .
$$

Suppose that $f: \mathbb{N} \rightarrow \mathbb{N}$ is total recursive with $f m r \forall x^{0} \operatorname{MP}^{\vee}\left(\alpha_{x}, \beta_{x}\right)$. Then, as in the proof of Lemma 1, one gets that

$$
(*)\left\{\begin{aligned}
\forall x(\{x\}(x) & \simeq 1 \vee\{x\}(x) \simeq 0 \rightarrow \\
{[f(x)} & =0 \rightarrow\{x\}(x) \simeq 1] \wedge[f(x) \neq 0 \rightarrow\{x\}(x) \simeq 0]) .
\end{aligned}\right.
$$


Let $e$ be a code of the total recursive function $s g \circ f$. Then $\{e\}(e)$ is defined with values $\leq 1$, i.e. $\{e\}(e) \simeq 1 \vee\{e\}(e) \simeq 0$ and so by $(*)$

$$
\{e\}(e) \simeq 0 \leftrightarrow\{e\}(e) \simeq 1,
$$

which is a contradiction.

Analogously to Theorem 2, Lemmas 4 and 5 imply

Theorem 6. Let $\Gamma$ be any set of $\exists$-free axioms which are true in $\mathcal{S}^{\omega}$ plus any $\mathcal{S}^{\omega}$-valid axioms whose modified realizability only requires total computable functions (type degree $\leq 1)$. Then

$$
\mathrm{E}-\mathrm{HA}^{\omega}+\mathrm{AC}+\mathrm{IP}_{e f}^{\omega}+\Gamma \nvdash \mathrm{MP}^{\vee-} .
$$

Corollary 7. E-HA ${ }^{\omega}+\Delta_{1}^{0}$-CA $\nvdash \mathrm{MP}^{\vee-}$.

Proof: By Theorem 6 and Proposition 1.

Acknowledgement: The author is grateful to Makoto Fujiwara for bringing the question addressed in this note to his attention.

\section{References}

[1] Akama, Y., Berardi, S., Hayashi, S., Kohlenbach, U., An arithmetical hierarchy of the law of excluded middle and related principles. Proc. of the 19th Annual IEEE Symposium on Logic in Computer Science (LICS'04), pp. 192-201, IEEE Press (2004).

[2] Ishihara, H., Markov's principle, Church's thesis and Lindelöf's theorem. Indag. Mathem., N.S. 4, pp. 321-325 (1993).

[3] Kohlenbach, U., Applied Proof Theory: Proof Interpretations and their Use in Mathematics. Springer Monographs in Mathematics. xx+536pp., Springer Heidelberg-Berlin, 2008 .

[4] Troelstra, A.S. (ed.) Metamathematical investigation of intuitionistic arithmetic and analysis. Springer Lecture Notes in Mathematics 344 (1973). 